\title{
Comment on "Robustness of proxy-based climate field reconstruction methods" by Michael E. Mann et al.
}

\author{
Jason E. Smerdon, ${ }^{1}$ J. Fidel González-Rouco, ${ }^{2}$ and Eduardo Zorita ${ }^{3}$ \\ Received 27 October 2007; revised 8 February 2008; accepted 17 July 2008; published 19 September 2008.
}

Citation: Smerdon, J. E., J. F. González-Rouco, and E. Zorita (2008), Comment on "Robustness of proxy-based climate field reconstruction methods" by Michael E. Mann et al., J. Geophys. Res., 113, D18106, doi:10.1029/2007JD009542.

[1] Mann et al. [2007a] (hereafter M07a) test the climate field reconstruction (CFR) method known as regularized expectation maximization (RegEM) using pseudoproxies derived from millennial simulations of past climate. These simulations were derived from two General Circulation Models (GCMs) driven with natural and anthropogenic forcings: the National Center for Atmospheric Research Climate System Model (CSM) [Boville et al., 2001] and the Hamburg Atmosphere-Ocean Coupled Circulation Model (ECHO-g) [Legutke and Voss, 1999]. There has been some discussion about the amplitude of millennial changes in simulations from these two GCMs [Goosse et al., 2005; Mann et al., 2005; Osborn et al., 2006; González-Rouco et $a l ., 2006]$, particularly with regard to how it may impact the assessment of CFR methods in pseudoproxy experiments [Mann et al., 2005; Mann, 2007; Zorita et al., 2007; Mann et al., 2007a, 2007b].

[2] Mann et al. [2005] argued that pseudoproxy tests performed with the ECHO-g ERIK1 millennial integration [von Storch et al., 2004] were jeopardized by the fact that the simulation suffered from an initialization drift in its early centuries and that it did not include aerosol forcings during the 20th century; by contrast, the CSM integration used by Mann et al. [2005] was drift corrected and included 20th-century aerosol forcings [Ammann et al., 2007]. Subsequent studies and discussions [Zorita et al., 2007; Mann et al., 2007a, 2007b] have clearly demonstrated, however, that differences between the ERIK1 and CSM model integrations have little impact on reported pseudoproxy tests. Nevertheless, there is an obvious importance to these discussions that goes beyond the context of pseudoproxy experiments and it is essential to accurately represent the similarities and differences between the two sets of model results within the public record. The purpose of this comment is to point out that $\mathrm{M} 07 \mathrm{a}$ have misrepresented the mean Northern Hemisphere $(\mathrm{NH})$ time series of the ERIK1 simulation and an accurate comparison between the CSM and ECHO-g simulations is therefore compromised.

\footnotetext{
${ }^{1}$ Lamont-Doherty Earth Observatory, Columbia University, Palisades, New York, USA.

${ }^{2}$ Departamento de Física de la Tierra, Astronomía y Astrofísica II, Universidad Complutense, Madrid, Spain.

${ }^{3}$ GKSS Research Center, Geesthacht, Germany.

Copyright 2008 by the American Geophysical Union. 0148-0227/08/2007JD009542
}

Furthermore, this mistake affects the verification statistics that have been reported for the RegEM CFR method, as well as exaggerate the impact of detrending on RegEM reconstructions that use the ERIK1 simulation.

[3] In Figure 1a we plot our calculation of the NH time series for the ECHO-g ERIK1 millennial integration, as well as the ERIK1 time series plotted by M07a in their Figures 4 and 5 and auxiliary material Figure S9. As in M07a, our calculated time series in Figure 1a has been referenced to the period 1900-1980 C.E. and has been filtered with the same decadal low-pass filter used by M07a. Also consistent with M07a, we calculate the area-weighted result for the restricted subset of the $5^{\circ}$ grid in the NH over which Mann and Rutherford [2002] report there to be sufficient coverage of instrumental observations since the mid-19th century. The straightforward interpretation of the comparison in Figure 1a is that the ERIK1 time series from M07a depicts considerably more variability in the simulation than exists in the time series that we have calculated.

[4] Upon the original drafting of this comment, we were not able to identify the source of the discrepancy reported herein. In their reply, however, Rutherford et al. [2008] associate the differences with the interpolation method used to transform the T30 Gaussian grid for the ECHO-g simulation $\left(\sim 3.75^{\circ}\right.$ latitude-longitude resolution) to a $5^{\circ}$ latitude-longitude grid; this discrepancy does not affect the CSM data, which were interpolated by a different means [Rutherford et al., 2008]. We note here that our results in Figure 1 are calculated for a $5^{\circ} \times 5^{\circ}$ interpolation of the original ECHO-g data using a bilinear interpolation scheme. While there are many interpolation methods from which to choose, it is important to note that the chosen method should yield an interpolated grid that closely compares to the originally resolved data. In Figure $1 \mathrm{~b}$ we compare the area-weighted $\mathrm{NH}$ mean time series calculated from the originally resolved ERIK1 data and our interpolated $5^{\circ} \times 5^{\circ}$ grid. The two time series are essentially identical, as expected for a viable interpolation method and a final grid resolution that is not significantly different from the original. A similar result would presumably not be achieved for the interpolated grid used by M07a. The simple demonstration in Figure $1 \mathrm{~b}$ therefore suggests that our interpolation is a least better than that used by M07a, if not implicating that the latter method and resulting grid are plainly incorrect.

[5] Perhaps the most important implication of the comparison shown in Figure 1a is the fact that the M07a ERIK1 

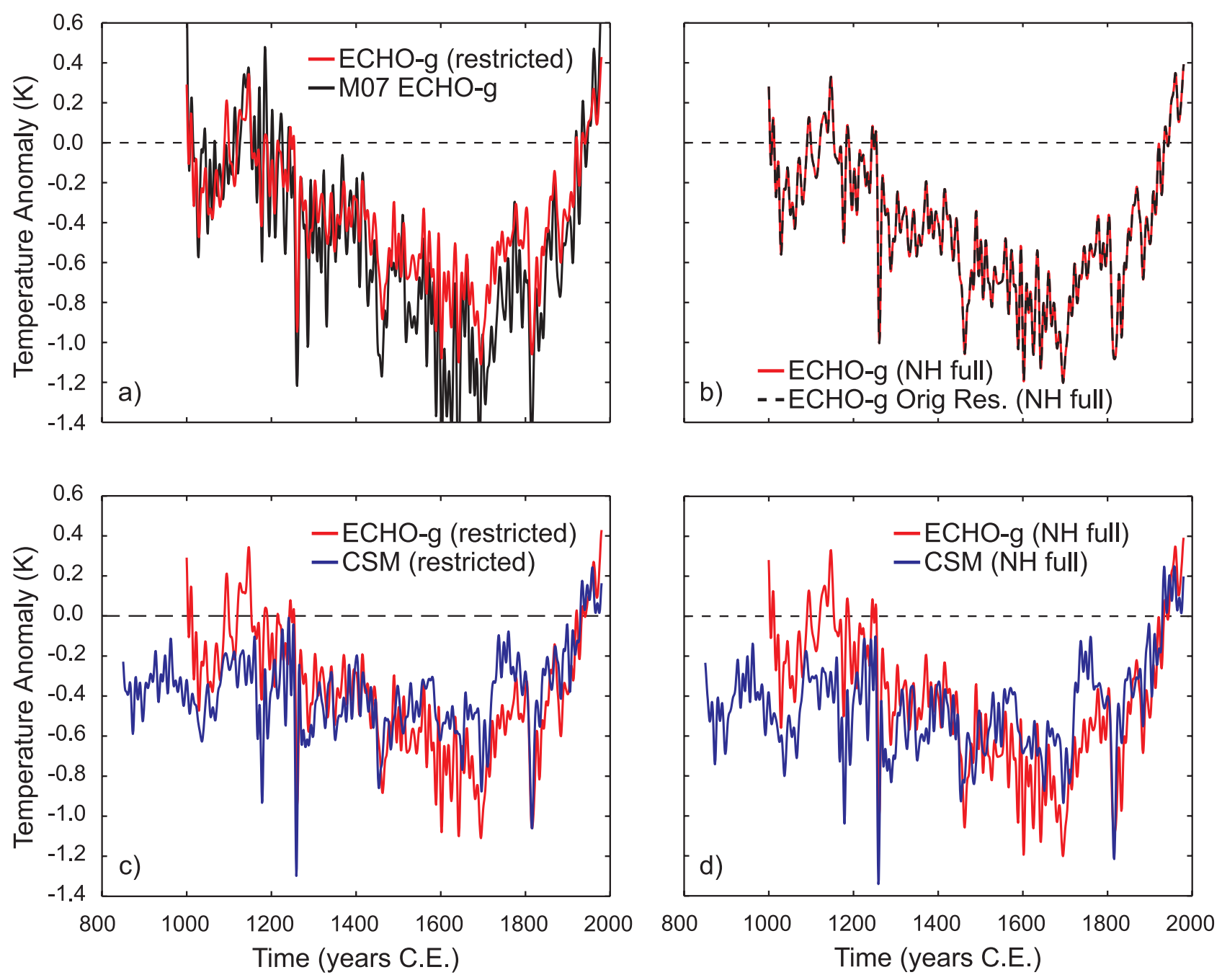

Figure 1. Comparison of: (a) the actual time series for the ECHO-g ERIK1 simulation for the restricted 669 grid-cell region of the NH and the time series shown in M07a; (b) the full area-weighted NH mean time series using the original ECHO-g grid $\left(\sim 3.75^{\circ}\right.$ grid point distance) and the interpolated $5^{\circ}$ latitudelongitude grid used in all other representations within this text; (c) the correct time series for the ECHO-g ERIK1 and CSM simulations for the restricted 669 grid-cell region in the $\mathrm{NH}$; and (d) the correct time series for the ECHO-g ERIK1 and CSM simulations for all grid cells in the NH. All time series have been smoothed with the same decadal low-pass filter used in M07a and referenced to the 1900-1980 C.E. interval. Figure 1 should be compared with M07a Figures 4, 5, and S9.

time series displays a larger amount of variability than actually exists in the simulation (for the purpose of comparison, Figure 1a is plotted over the temperature range used for the CSM data in M07a and therefore does not show a few of the largest temperature anomalies in the M07a ERIK1 series). This misrepresentation of the ERIK1 time series therefore depicts larger differences between the CSM and ECHO-g simulations. In Figures $1 \mathrm{c}$ and $1 \mathrm{~d}$ we plot the area-weighted $\mathrm{NH}$ time series for the CSM and ERIK1 millennial integrations, using the restricted (669 grid cells) and full (1296) NH domains, respectively. The plots indicate that there are indeed differences between the two integrations, particularly in the early centuries when ERIK1 is warmer. The overall ranges of the mean time series are not that different, however, and they can be easily plotted with the same vertical scaling. By contrast, Figures 4 and 5 of M07a plot the CSM ranges because their calculated ERIK1 time series ranges from $-1.51 \mathrm{~K}$ to $0.97 \mathrm{~K}$ (far exceeding the range of the correct time series from -0.99 to $0.55 \mathrm{~K}$ ).

[6] In conclusion, we note that the technical issue discussed herein does not impact the basic conclusions of M07a regarding the performance of the RegEM CFR method. Nevertheless, several important implications should be noted. First, the verification statistics in Table 1 of $\mathrm{M} 07 \mathrm{a}$ are weakened when an appropriate interpolation scheme is adopted; these numbers should be corrected to avoid hindering efforts to reproduce the M07a results and to provide consistent evaluations of the RegEM CFR method across different model integrations. Second, the effect of detrending the target data, while clearly significant, is visually amplified in Figure 5b of M07a because the target $\mathrm{NH}$ time series will not range as far from zero as is presently shown. We expect that a corrected figure for the ECHO-g 
data would look more similar to the result shown in the M07a Figure 5a for the CSM result. Finally, we reiterate that the misrepresentation noted herein depicts a notably larger variability in the ERIK1 simulation. Given that the range of variability in this ECHO-g integration has been the subject of much criticism in previous publications by Mann and coauthors [e.g., Mann et al., 2005; Mann, 2007; Mann et al., 2007b], it is important that accurate representations of these simulations are maintained. Contrary to what is implied by the time series shown in M07a, Figure 1 and several previous publications [e.g., Goosse et al., 2005; Zorita et al., 2007] clearly demonstrate that the ECHO-g and CSM simulations exhibit a comparable mean $\mathrm{NH}$ temperature range. Furthermore, we would be remiss if we did not point out that this same misrepresentation of the ERIK1 mean NH time series appears in several other publications of which we are aware [Mann, 2007; Mann et $a l ., 2007 \mathrm{~b}]$. It is essential that these misrepresentations are documented in order to avoid equivocal interpretations of the ECHO-g data and related results.

[7] Acknowledgments. This research was supported by the U.S. National Oceanic and Atmospheric Administration by grant NA07OAR4310060 (J.E.S.), by project SPECT (CGL2005-06097/CLI) and the RyC Program, by the Spanish MEC (J.F.G.-R), and by the European project Millennium European Climate (contract 017008 GOCE; E.Z.). LDEO contribution 7194

\section{References}

Ammann, C. M., F. Joos, D. S. Schimel, B. L. Otto-Bliesner, and R. A. Tomas (2007), Solar influence on climate during the past millennium: Results from transient simulations with the NCAR Climate System Model, Proc. Natl. Acad. Sci. U. S. A., 104, 3713-3718, doi:10.1073/ pnas.0605064103.

Boville, B. A., J. T. Kiehl, P. J. Rasch, and F. O. Bryan (2001), Improvements to the NCAR CSM-1 for transient climate simulations, J. Clim., 14, 164-179, doi:10.1175/1520-0442(2001)014<0164:ITTNCF> 2.0.CO;2.

González-Rouco, J. F., H. Beltrami, E. Zorita, and H. von Storch (2006), Simulation and inversion of borehole temperature profiles in surrogate climates: Spatial distribution and surface coupling, Geophys. Res. Lett., 33, L01703, doi:10.1029/2005GL024693.
Goosse, H., T. J. Crowley, E. Zorita, C. M. Ammann, H. Renssen, and E. Driesschaert (2005), Modelling the climate of the last millennium: What causes the differences between simulations?, Geophys. Res. Lett., 32, L06710, doi:10.1029/2005GL022368.

Legutke, S., and R. Voss (1999), The Hamburg Atmosphere-Ocean Coupled Circulation Model ECHO-g, Tech. Rep. 18, Dtch. Klimarechenzentrum, Hamburg.

Mann, M. E. (2007), Climate over the past two millennia, Annu. Rev. Earth Planet. Sci., 35, 111-136, doi:10.1146/annurev.earth.35.031306.140042.

Mann, M. E., and S. Rutherford (2002), Climate reconstructions using "Pseudoproxies," Geophys. Res. Lett., 29(10), 1501, doi:10.1029/ 2001GL014554

Mann, M. E., S. Rutherford, E. Wahl, and C. Amman (2005), Testing the fidelity of methods used in proxy-based reconstructions of past climate, J. Clim., 18, 4097-4107, doi:10.1175/JCLI3564.1.

Mann, M. E., S. Rutherford, E. Wahl, and C. Ammann (2007a), Robustness of proxy-based climate field reconstruction methods, J. Geophys. Res., 112, D12109, doi:10.1029/2006JD008272.

Mann, M. E., S. Rutherford, E. Wahl, and C. Ammann (2007b), Reply to comments on 'Testing the fidelity of methods used in proxy-based reconstructions of past climate' by Zorita et al., J. Clim., 20, 3699-3703, doi:10.1175/JCLI4172.1.

Osborn, T., S. C. B. Raper, and K. R. Briffa (2006), Simulated climate change during the last 1000 years: Comparing the ECHO-g general circulation model with the MAGICC simple climate model, Clim. Dyn., 27, 185-197, doi:10.1007/s00382-006-0129-5.

Rutherford, R., M. E. Mann, E. Wahl, and C. Ammann (2008), Reply to Comment on 'Testing the fidelity of methods used in proxy-based reconstructions of past climate' by Mann et al., J. Clim., 20, 3699-3703, doi:10.115/JCLI4172.1.

von Storch, H., E. Zorita, Y. Dimitriev, F. González-Rouco, and S. Tett (2004), Reconstructing past climate from noisy data, Science, 306, 679-682, doi:10.1126/science. 1096109.

Zorita, E., F. González-Rouco, and H. von Storch (2007), Comments to 'Testing the fidelity of methods used in proxy-based reconstructions of past climate' by Mann et al., J. Clim., 20, 3693-3698, doi:10.1175/ JCLI4171.1.

J. F. González-Rouco, Departamento de Física de la Tierra, Astronomía y Astrofísica II, Universidad Complutense, Madrid E-28040, Spain. (fidelgr@fis.ucm.es)

J. E. Smerdon, Lamont-Doherty Earth Observatory, Columbia University, Palisades, NY 10964, USA. (jsmerdon@1deo.columbia.edu)

E. Zorita, GKSS Research Center, Max Planck Strasse 1, D-21502 Geesthacht, Germany. (Eduardo.Zorita@gkss.de) 